\title{
A REMARK ON A DECOMPOSITION SPACE OF BING
}

\author{
R. P. HUNTER
}

The following question has been raised by several authors: If $X$ and $Y$ are two continua whose cartesian product admits a continuous associative multiplication with identity, must $X$ and $Y$ both admit such multiplications? We remark here, that a certain decomposition space defined by Bing yields a negative answer.

Let $\bar{B}$ denote the space described in 8 of [1]. It is a certain upper semicontinuous decomposition of $S^{3}$ each of whose elements is an arc or a point. It is shown in [1] that $\bar{B} \times S^{1}$ is homeomorphic with $S^{3} \times S^{1}$. Now $S^{3} \times S^{1}$ admits the structure of a topological group. Hence we need only show that $\bar{B}$ cannot admit the structure of a topological semigroup with identity. We note first the following

LEMMA. Let $D$ and $T$ be two compact connected topological semigroups with identities. If $D \times T$ is topologically $a$ manifold then $D$ and $T$ are already Lie groups.

Using coordinatewise multiplication, we see that $D \times T$ is a semigroup with identity. Then however, [3], it is well known that $D \times T$ is already a group. Since $D \times T$ can then have only one idempotent it is immediate that $D$ has only one idempotent. But a compact semigroup with a unique idempotent which is an identity must already be a group, [2]. Hence $D$ is a topological group and, being locally connected and finite dimensional, is a Lie group. Likewise, $T$ is already a Lie group.

Since $\bar{B} \times S^{1}$ is a manifold, $S^{3} \times S^{1}$, we see that were $\bar{B}$ to be a semigroup with identity it would be a Lie group. Since $\bar{B}$ is not a manifold the result follows.

\section{BIBLIOGRAPHY}

1. R. H. Bing, The Cartesian product of a certain non-manifold and a line is $E_{4}$, Ann. of Math. (2) 70 (1959), 399-412.

2. R. J. Koch and A. D. Wallace, Maximal ideals in compact semigroups, Duke Math. J. 21 (1954), 681-686.

3. A. D. Wallace, The structure of topological semigroups, Bull. Amer. Math. Soc. 61 (1955), 95-112.

The University of Georgia

Received by the editors July 17, 1961. 